\title{
Classification of Different Blueberry Cultivars by Analysis of Physical Factors, Chemical and Nutritional Ingredients, and Antioxidant Capacities
}

\author{
Juan Song $\mathbb{D}^{1},{ }^{1}$ Qiong Shi $\mathbb{D},{ }^{2}$ Si-Min Yan, ${ }^{3}$ Hai-Yan Fu $\mathbb{D}^{2},{ }^{2}$ Si-Zhan Wu $\mathbb{D}^{1},{ }^{1}$ and Lu Xu ${ }^{1}$ \\ ${ }^{1}$ College of Material and Chemical Engineering, Tongren University, Tongren 554300, Guizhou, China \\ ${ }^{2}$ The Modernization Engineering Technology Research Center of Ethnic Minority Medicine of Hubei Province, \\ College of Pharmacy, South-Central University for Nationalities, Wuhan 430074, China \\ ${ }^{3}$ Shanghai Institute of Quality Inspection and Technical Research, Shanghai 201114, China
}

Correspondence should be addressed to Hai-Yan Fu; fuhaiyan@mail.scuec.edu.cn and Lu Xu; lxchemo@163.com

Received 27 March 2020; Revised 18 August 2020; Accepted 29 August 2020; Published 26 September 2020

Academic Editor: Encarna Aguayo

Copyright ( 92020 Juan Song et al. This is an open access article distributed under the Creative Commons Attribution License, which permits unrestricted use, distribution, and reproduction in any medium, provided the original work is properly cited.

\begin{abstract}
Blueberry fruits of different cultivars are featured with different quality indices. In this work, three types of quality factors, including 6 physical parameters, 12 chemical and nutritional components, and 3 antioxidant indices, were measured to compare and classify blueberry fruits from 12 different cultivars in China. Using the autoscaled data of quality factors, unsupervised principal component analysis was performed for exploratory analysis of intercultivar differences and the influences of quality factors. A supervised classification method, partial least squares discriminant analysis (PLSDA), was combined with the global particle swarm optimization algorithm (PSO) and two multiclass strategies, one-versus-rest (OVR) and one-versus-one (OVO), to select discriminative quality factors and develop classification models of the 12 cultivars. As a result, OVO-PLSDA with 8 quality factors could achieve the classification accuracy of 0.915 . This study will provide new insights into the quality variations and key factors among different blueberry cultivars.
\end{abstract}

\section{Introduction}

Blueberry, a native American cultivar, has been widely cultivated around the world [1]. It is a very popular fruit due to its pleasant flavor, high nutritional value, and healthy effects $[2,3]$. Fresh blueberry fruits are rich in various nutritional ingredients, such as anthocyanins, polyphenols, flavonoids, polysaccharides, vitamins, minerals, and dietary fibres, [4]. Moreover, modern scientific experiments have revealed many of its functional activities, such as antioxidant, antimicrobial, antihypertensive, anti-inflammatory, and neuroactive properties, and its ability to prevent obesity, diabetes, cancer, and other chronic diseases [5-10]. Besides being consumed as fresh fruits, blueberries are also widely used to produce natural extracts, blueberry wine, beverages, jams, preserved fruits, and food ingredients or additives.
The cultivation of blueberry in China started in the mid1980 s and began to be popularized in the early 21 st century. At present, the main cultivation area has amounted to about $3500 \mathrm{hm}^{2}$ around Northeast and North China, Jiangsu, Zhejiang, Liaodong Peninsula, and Southwest and South China [11]. According to an incomplete survey, over 100 different cultivars of blueberries have been introduced and bred in China, among which about 10-15 cultivars are important in the domestic market [12].

The physical and chemical quality factors, the nutritional ingredients, and functional activities of blueberries depend largely on the specific cultivars and cultivation conditions. Many sensory indices (such as hardness, brittleness, and chewiness) of fruits are closely related to texture and physical factors, which directly affect their storage and transportation characteristics. They have become important indices for 
testing fruit quality and the primary factors for evaluating acceptability of freshly consumed fruits [13]. The levels of chemical indices and nutritional ingredients also play an important role in the flavor and nutritional value of blueberries, which have been intensively studied and compared among different cultivars and producing areas [14]. Among its various functional activities, the antioxidant capacity and substances, such as polyphenols (especially anthocyanins) and flavonoids, are also associated with many other functional activities and have been compared with blueberries from different cultivars, geographical origins, and with different postharvest processing methods [15].

Statistical and chemometrics have been widely used to reveal the contributions of multiple variables in complex chemical systems [16-21]. At present, the studies on blueberry quality mainly focus on one or several indicators, but few studies have been performed yet on comprehensive evaluations of physical, chemical, and nutritional quality factors among different cultivars of blueberries [22-24]. The objective of this work was to study the quality variations among some major blueberry cultivars by a fusion analysis of some physical and chemical factors, nutritional ingredients, and antioxidant abilities. In order to reveal the key factors among different blueberry cultivar, besides the unsupervised principal component analysis (PCA), the supervised partial least squares discriminant analysis (PLSDA) was also used to develop multiclass classification models using feature sets selected by the global particle swarm optimization (PSO) algorithm [25-28].

\section{Materials and Methods}

2.1. Blueberry Samples. Mature blueberry fruit samples $(N=366)$ of 12 different cultivars were provided by several local blueberry orchards in Huaining, Anhui province. The blueberry fruit was harvested in 3 days after attaining the maximum blue color. After harvesting, the blueberries were packed in fresh-keeping boxes and placed in $4^{\circ} \mathrm{C}$ incubators, which were transported to the lab on the second day. Intact fruits with uniform size and color were selected, and their quality indexes were measured. Fresh fruits were washed and dried, packed and sealed, and frozen at $-18^{\circ} \mathrm{C}$ to be used for determination of physicochemical indexes. The detailed information about sample size, cultivar, and sources are listed in Table 1.

2.2. Quality Analysis of Blueberry Fruit. In this work, a set of 21 quality factors, including 6 physical factors, 12 chemical and nutritional components, and 3 antioxidant indices, were determined for the collected blueberry fruit. The 21 quality factors are listed in Table 2.

2.3. Measurements of Physical Factors. For each sample, 10 blueberry fruits were randomly selected, and $L *$ and hardness were measured at 3 sites along the equatorial line. The measurement of lightness value $(L *)$ was performed using a CR-400 Chroma Meter (Minolta, Osaka, Japan). The hardness value was determined using the method by $\mathrm{Hu}$ et al. with a TA.XTplus texture analyser (Stable Micro
Systems, England) [29]. The average single fruit weight, shape index (the ratio of maximum height to width), and specific gravity were also measured on 10 randomly selected fruits. To measure the juice yield, fruits $(20 \mathrm{~g})$ were beaten and centrifuged at $6000 \mathrm{r} / \mathrm{min}$ for $15 \mathrm{~min}$ to obtain the upper juice.

2.4. Determination of Chemical Factors and Nutritional Ingredients. The total soluble solid (in ${ }^{\circ}$ Brix) was analyzed using a PAL-1 Digital Hand-Held Pocket Refractometer (Atago, Japan). The $\mathrm{pH}$ value was measured on blueberry pulp using a PB-10 pH meter (Sartorius, Germany). The titratable acidity (TA) value was analyzed using an indicator method for acidbase titration [30]. The level of vitamin C (ascorbic acid) was determined by the 2,4-dinitrophenylhydrazine colorimetric method with a U-3900 ultraviolet-visible spectrophotometer (Hitachi, Tokyo, Japan) [31]. The contents of total phenols (TP) were analyzed using the Folin-Ciocalteu method [32]. The standard curve was made using pyrogallic acid (in ethanol), and TP content was computed as gallic acid equivalents per $100 \mathrm{~g}$ fresh weight (mg GAE/100g FW). The analysis of total flavonoids (TF) was performed using the $\mathrm{AlCl}_{3}$ colorimetric method [33]. TF content was expressed as catechin equivalents per $100 \mathrm{~g}$ fresh weight $(\mathrm{mg} \mathrm{CE} / 100 \mathrm{~g} \mathrm{FW})$. The total soluble sugar was determined using the modified anthrone-sulfuric acid method [34]. The level of reducing sugar was analyzed using the direct titration method of copper tartrate solution [35]. The moisture was determined by the direct drying method [36]. The ash content was analyzed by burning and weighing [37]. Protein determination was performed using the classical Kjeldahl method [38]. The contents of anthocyanins were determined by the differential $\mathrm{pH}$ method [39].

2.5. Antioxidant Analysis. Three different antioxidant indices were measured to compare the antioxidant capacities of blueberries. The scavenging capacity of 2, 2-diphenyl-1picrylhydrazyl (DPPH) and hydroxyl radicals and the ferric reducing antioxidant power (FRAP) values were determined following the procedures described for blueberry samples $[5,40,41]$.

2.6. Chemometrics Data Analysis. Considering the scale variations in different quality factors, each factor was autoscaled, namely, the values were made to have a zero mean and a standard deviation of 1 . For exploratory analysis of the data, unsupervised principal component analysis (PCA) was performed to show the class distributions of blueberry [42]. The DUPLEX algorithm was performed to divide the data of each class into training and test objects, which were combined to generate the final training and test sets [43].

Partial least squares discriminant analysis (PLSDA) was used to develop two-class classification models [44-47]. To tackle the multiclass problems in this work, two chemometrics strategies, one-versus-rest (OVR) and one-versusone (OVO), were performed and compared to develop a set of binary PLSDA classifiers [48, 49]. 
TABLE 1: Blueberry samples of 12 different cultivars collected in Huaining (Anhui province).

\begin{tabular}{|c|c|c|}
\hline $\begin{array}{l}\text { Class } \\
\text { number }\end{array}$ & Sample size & Cultivar and orchard \\
\hline 1 & $31(19,12)^{\mathrm{a}}$ & O’Neal, Huangdun \\
\hline 2 & $30(20,10)$ & Sharpblue, Huangdun \\
\hline 3 & $31(19,12)$ & $\begin{array}{c}\text { Powderblue, Qinghe, and } \\
\text { Huangdun }\end{array}$ \\
\hline 4 & $30(20,10)$ & Climax, Sanqiao \\
\hline 5 & $30(20,10)$ & Premier, Xiushan \\
\hline 6 & $32(20,12)$ & Gardenblue, Xiaoshi \\
\hline 7 & $30(20,10)$ & Brightwell, Sanqiao \\
\hline 8 & $30(20,10)$ & Baldwin, Gongling \\
\hline 9 & $31(19,12)$ & Duxiu, Huangdun \\
\hline 10 & $30(20,10)$ & Blomidon, Gaohe, and Huangdun \\
\hline 11 & $29(19,10)$ & Bluecrop, Sanqiao \\
\hline 12 & $32(20,12)$ & Bluegold, Qinghe \\
\hline Sum & $366(236,130)$ & \\
\hline
\end{tabular}

${ }^{\mathrm{a}}$ The numbers of the training and test objects for classification selected by the DUPLEX algorithm.

TABLe 2: The 21 quality factors measured for blueberry fruit.

\begin{tabular}{|c|c|c|}
\hline No. & Factor & Type \\
\hline 1 & Lightness value $\left(L^{*}\right)$ & Physical \\
\hline 2 & Hardness & Physical \\
\hline 3 & Average single fruit weight & Physical \\
\hline 4 & Shape index & Physical \\
\hline 5 & Specific gravity & Physical \\
\hline 6 & Juice yield & Physical \\
\hline 7 & Total soluble solid & $\begin{array}{c}\text { Chemical and } \\
\text { nutritional }\end{array}$ \\
\hline 8 & $\mathrm{pH}$ & $\begin{array}{c}\text { Chemical and } \\
\text { nutritional }\end{array}$ \\
\hline 9 & Titratable acidity (TA) & $\begin{array}{l}\text { Chemical and } \\
\text { nutritional }\end{array}$ \\
\hline 10 & Vitamin C & $\begin{array}{l}\text { Chemical and } \\
\text { nutritional }\end{array}$ \\
\hline 11 & Total phenols (TP) & $\begin{array}{c}\text { Chemical and } \\
\text { nutritional }\end{array}$ \\
\hline 12 & Total flavonoids (TF) & $\begin{array}{c}\text { Chemical and } \\
\text { nutritional }\end{array}$ \\
\hline 13 & Total soluble sugar & $\begin{array}{c}\text { Chemical and } \\
\text { nutritional }\end{array}$ \\
\hline 14 & Reducing sugar & $\begin{array}{c}\text { Chemical and } \\
\text { nutritional }\end{array}$ \\
\hline 15 & Moisture & $\begin{array}{c}\text { Chemical and } \\
\text { nutritional }\end{array}$ \\
\hline 16 & Ash & $\begin{array}{c}\text { Chemical and } \\
\text { nutritional }\end{array}$ \\
\hline 17 & Proteins & $\begin{array}{c}\text { Chemical and } \\
\text { nutritional }\end{array}$ \\
\hline 18 & Anthocyanins & $\begin{array}{c}\text { Chemical and } \\
\text { nutritional }\end{array}$ \\
\hline 19 & $\begin{array}{l}\text { Scavenging capacity of 2,2-diphenyl-1- } \\
\text { picrylhydrazyl (DPPH) radical }\end{array}$ & Antioxidant \\
\hline 20 & Scavenging capacity of hydroxyl radical & Antioxidant \\
\hline 21 & $\begin{array}{l}\text { Ferric reducing antioxidant power } \\
\text { (FRAP) }\end{array}$ & Antioxidant \\
\hline
\end{tabular}

In order to probe and reveal the key quality factors reflecting the cultivar variations of blueberry, the global particle swarm optimization (PSO) algorithm was used to select the most discriminative feature sets [28]. PSO can imitate the social behavior of bird flocking where a population of particles or candidate solutions are improved iteratively to approach the best solution by combining random search and the best known solutions. PSO can be started with a population of random feasible solutions. In this work, 100 initial feasible solutions were randomly generated as strings of $0 \mathrm{~s}$ and $1 \mathrm{~s}$, where 0 and 1 represent the absence and presence of a quality factor, respectively. Discriminative feature sets were selected to obtain the lowest overall classification error rate of Monte Carlo cross validation (OCERMCCV) defined as

$$
\operatorname{OCERMCCV~}=\frac{\sum_{i=1}^{B} M_{i}}{\sum_{i=1}^{B} N_{i}}
$$

where $B$ is the number of random data splitting by MCCV; $M_{i}$ and $N_{i}$ are the number of misclassified objects and test objects for the $i$ th data splitting, respectively [50].

2.7. Software. All the data processing and chemometric algorithms were performed on MATLAB 7.0.1 (MathWorks, Sherborn, MA, USA). The DUPLEX algorithm was performed using the codes included in the TOMCAT toolbox [51]. All the other data analysis algorithms, including PCA, OVO, OVR, PLSDA, and PSO, were performed using selfcompiled MATLAB codes.

\section{Results and Discussion}

The ranges and standard deviations (SD) of the 21 quality factors of 12 blueberries are summarized in Table 3 where the raw data of quality factors have different scales. To illustrate the distribution of different classes, principal component analysis (PCA) was performed on the autoscaled data (Figure 1). The first two principal components (PCs) account for $87.59 \%$ of the total data variances. Projection of the 12 classes onto the first 2 PCs showed the variations among different cultivars of blueberries. The loadings of the first PC (Figure 1) indicated that the levels of 1 physical parameter (hardness) and 5 chemical and nutritional components (vitamin $\mathrm{C}$, total phenols, total flavonoids, proteins, and anthocyanins) contribute significantly to the class separation achieved by PC1. For the second PC (Figure 1), 5 parameters had important contributions, including 2 physical parameters (average single fruit weight and shape index), 2 chemical and nutritional components (titratable acidity and anthocyanins), and an antioxidant index (scavenging capacity of DPPH radical). Obviously, the level of anthocyanins was a key quality factor to discriminate different blueberries as it plays an important role in both PC1 and PC2.

Although PCA could obtain some separation of different blueberries, supervised methods were needed to achieve more accurate classification models based on key quality parameters. Therefore, multiclass classification models were developed using OVR-PLSDA and OVO-PLSDA models with subsets of key quality parameters selected by the PSO algorithm. To obtain representative training and test data sets, the DUPLEX 
TABLE 3: Ranges of quality parameters of blueberry fruits of different cultivars.

\begin{tabular}{|c|c|c|c|}
\hline No. & Parameters & Range of average & $\mathrm{SD}$ \\
\hline 1 & Lightness value $\left(L^{*}\right)$ & $27.33-36.18$ & 1.87 \\
\hline 2 & Hardness $(\mathrm{N})$ & $241.60-571.53$ & 112.36 \\
\hline 3 & Average single fruit weight (g) & $1.72-2.61$ & 0.29 \\
\hline 4 & Shape index & $0.72-0.97$ & 0.09 \\
\hline 5 & Specific gravity $\left(\mathrm{g} / \mathrm{cm}^{3}\right)$ & $0.97-1.19$ & 0.07 \\
\hline 6 & Juice yield (w/w\%) & $56.11-78.38$ & 7.85 \\
\hline 7 & Total soluble solid (w/w\%) & $7.86-12.21$ & 1.68 \\
\hline 8 & $\mathrm{pH}$ & $3.11-3.76$ & 0.24 \\
\hline 9 & Titratable acidity (TA) (w/w\%) & $0.33-0.81$ & 0.15 \\
\hline 10 & Vitamin C $(\mathrm{mg} / 100 \mathrm{~g} \mathrm{FW})$ & $18.36-93.25$ & 28.83 \\
\hline 11 & Total phenols (TP) (mg GAE/100 g FW) & $244.63-369.59$ & 43.11 \\
\hline 12 & $\begin{array}{l}\text { Total flavonoids (TF) } \\
(\mathrm{mg} \mathrm{CE} / 100 \mathrm{~g} \mathrm{FW})\end{array}$ & $279.39-531.11$ & 88.58 \\
\hline 13 & Total soluble sugar (w/w\%) & $6.88-11.32$ & 1.61 \\
\hline 14 & Reducing sugar (w/w\%) & $5.73-8.59$ & 1.07 \\
\hline 15 & Moisture (w/w\%) & $82.66-85.95$ & 1.37 \\
\hline 16 & $\operatorname{Ash}(\mathrm{w} / \mathrm{w} \%)$ & $0.21-0.36$ & 0.06 \\
\hline 17 & Proteins $(\mathrm{mg} / 100 \mathrm{~g} \mathrm{FW})$ & $388.29-729.39$ & 140.63 \\
\hline 18 & Anthocyanins $(\mathrm{mg} / 100 \mathrm{~g} \mathrm{FW})$ & $83.51-257.78$ & 55.96 \\
\hline 19 & Scavenging capacity of 2,2-diphenyl-1-picrylhydrazyl (DPPH) radical (mmol Trolox/100 g FW) & $1.15-3.55$ & 0.88 \\
\hline 20 & Scavenging capacity of hydroxyl radical (mmol Trolox/100 g FW) & $0.77-2.81$ & 0.69 \\
\hline 21 & Ferric reducing antioxidant power (FRAP) $\left(\mathrm{mmol} \mathrm{Fe}^{2+} / 100 \mathrm{~g} \mathrm{FW}\right)$ & $0.54-2.46$ & 0.74 \\
\hline
\end{tabular}

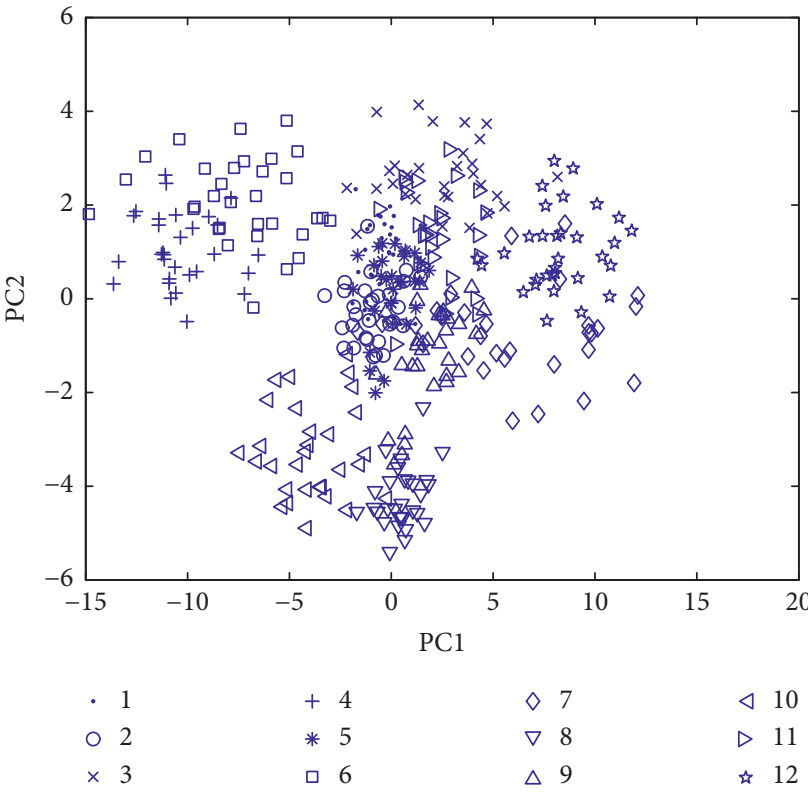

(a)

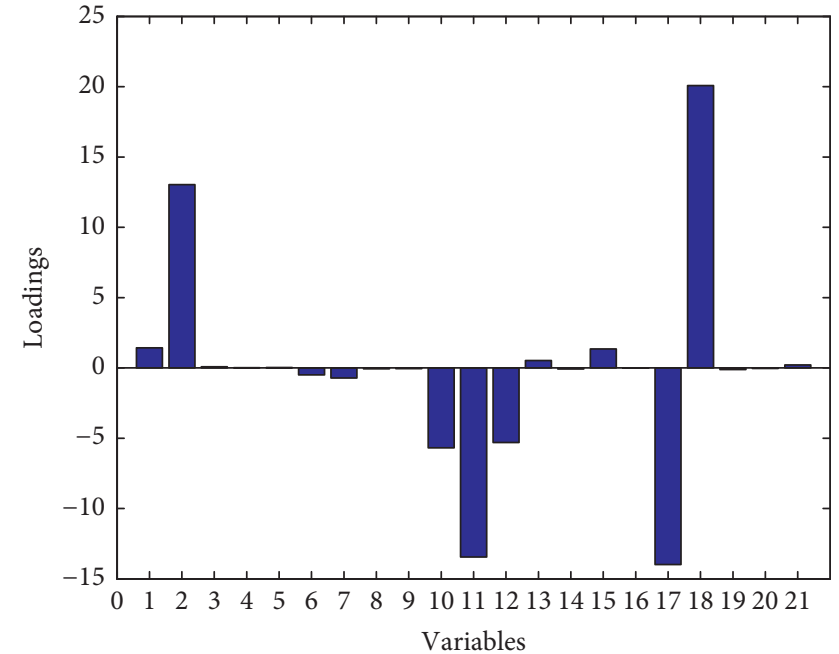

(b)

Figure 1: Continued. 


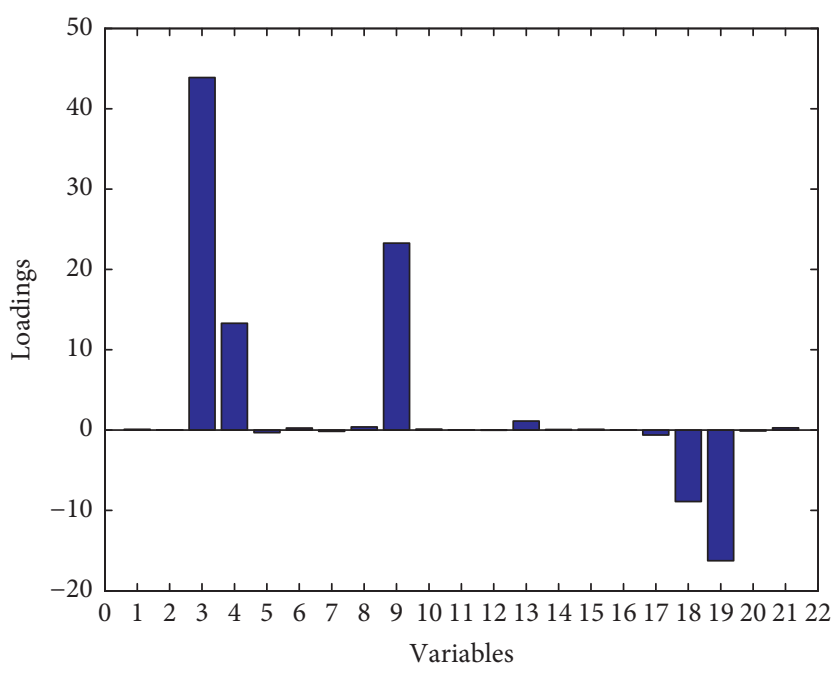

(c)

FIGURE 1: Scores (a) and loadings ( $\mathrm{b}$ and c) of the first two principal components (PCs) obtained by principal component analysis (PCA) of 21 quality factors for 12 cultivars of blueberries.

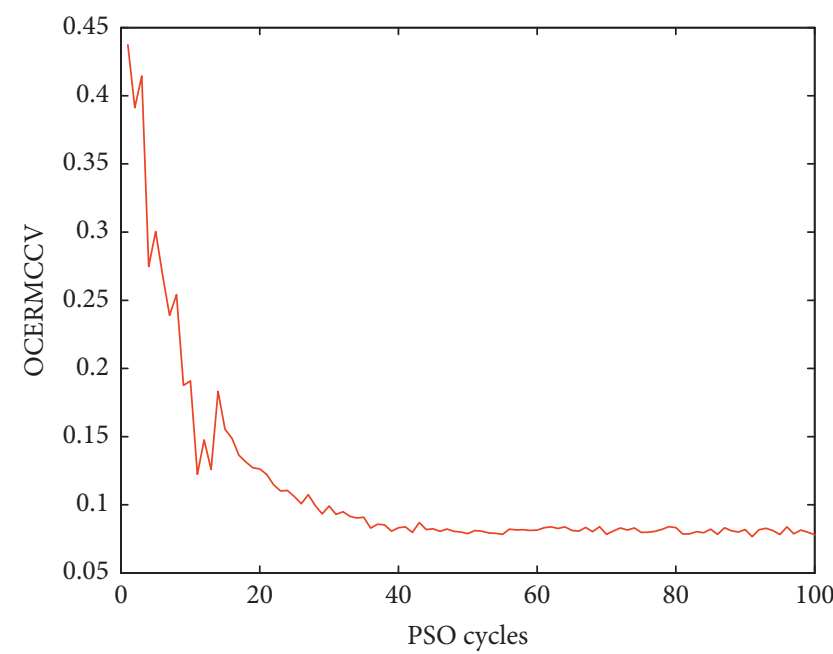

(a)

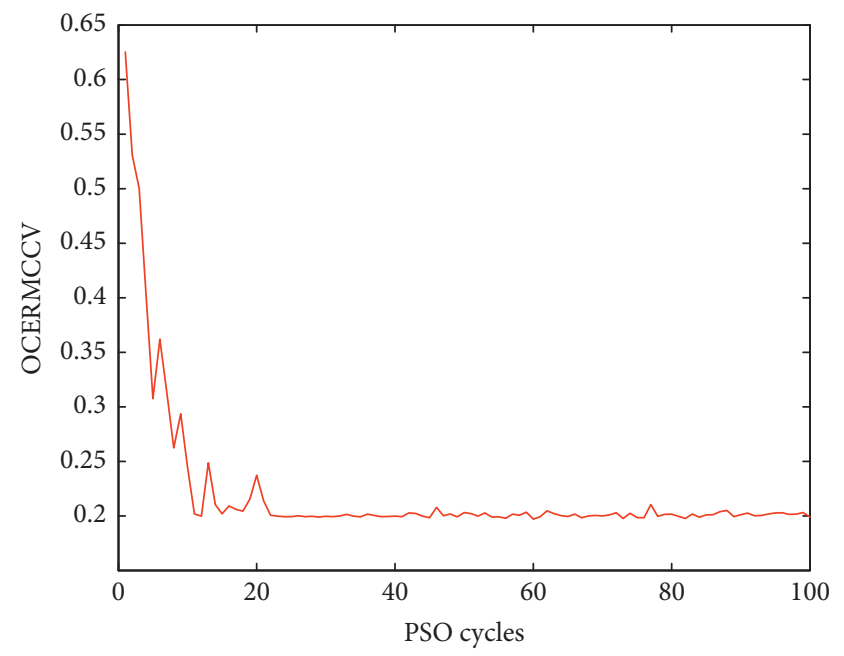

(b)

Figure 2: Trends of MCCV classification errors for 8-variable OVO-PLSDA and 10-variable OVR-PLSDA in 100 PSO cycles.

algorithm was performed on each of the 12 classes of blueberries to divide the measured data into training and test objects (Table 1). The training and test objects from each class were combined to generate the final training and test sets, including 236 and 130 objects, respectively.

'For both OVR-PLSDA and OVO-PLSDA, the number of significant latent variables (LVs) of each binary PLSDA submodel was determined using MCCV to obtain the lowest OCERMCCV. With different sizes (3-15) of parameter subsets, PSO was performed to search for the optimal subsets by minimizing the OCERMCCV. In this study, the number of data splitting of MCCV was 100 . For each data splitting, $80 \%$ of the training objects was used for model development, and $20 \%$ of the training objects was used for validation. For PSO, the algorithm was stopped when the value of objective function
(OCERMCCV) could not be reduced by $0.1 \%$ in the next cycle. The maximum total number of PSO cycles was set to be 100 , which to our knowledge was sufficient to solve the small-scale (21 variables to be selected) optimization problem in this work. To examine the optimization performances of PSO, a 100-cycle PSO was performed to search for the best subsets for the 8variable OVO-PLSDA and 10-variable OVR-PLSDA, and the lowest OCERMCCV for each cycle is shown in Figure 2. Though there were slight fluctuations of OCERMCCV, PSO could significantly reduce OCERMCCV with sufficient cycles, and the searching results were stable for both of the two methods.

The classification results of OVR-PLSDA and OVOPLSDA with selected subsets of quality factors are summarized in Table 4. In terms of OCERMCCV, for OVR- 
TABLE 4: Classification results of 12 cultivars of blueberries using optimized subsets of quality parameters.

\begin{tabular}{lcccc}
\hline Models & Subset size & Selected quality parameters $^{\mathrm{a}}$ & OCERMCCV $^{\text {Accuracy }^{\mathrm{b}}}$ \\
\hline \multirow{3}{*}{ OVR-PLSDA } & 10 & $1,2,4,7,8,11,12,17,18$, and 19 & 0.199 & 0.815 \\
& 12 & $1,3,4,7,9,10,11,12,17,18,19$, and 20 & 0.203 & 0.792 \\
\hline \multirow{3}{*}{ OVO-PLSDA } & 9 & $2,4,5,10,11,12,14,18$, and 19 & 0.209 & 0.078 \\
& 8 & $2,6,9,10,11,12,18$, and 19 & 0.093 & 0.915 \\
& 9 & $2,6,8,10,11,12,18,19$, and 21 & 0.104 & 0.900 \\
\hline
\end{tabular}

${ }^{a}$ The order of quality parameters is shown in Table $1{ }^{\mathrm{b}}$ The overall prediction accuracy of the 12 blueberries.

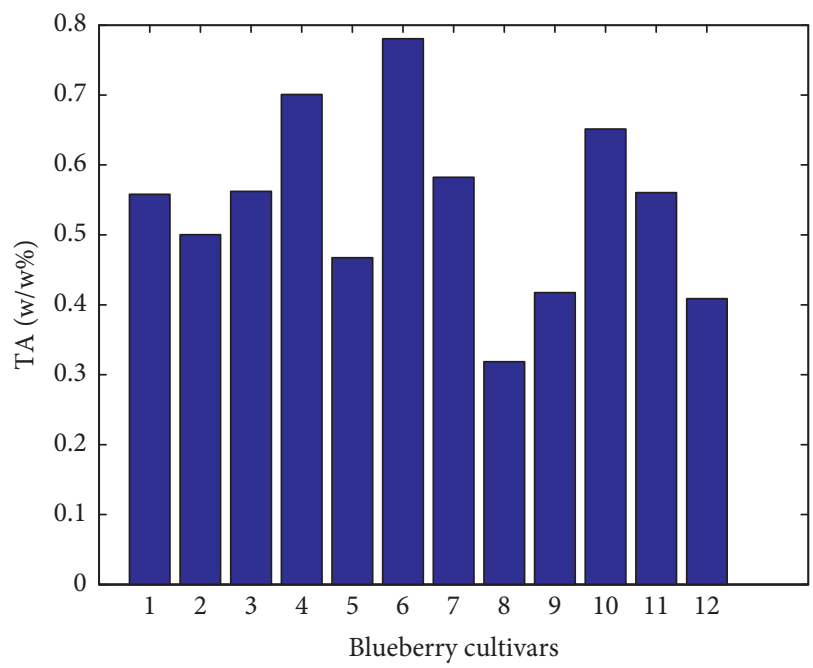

(a)

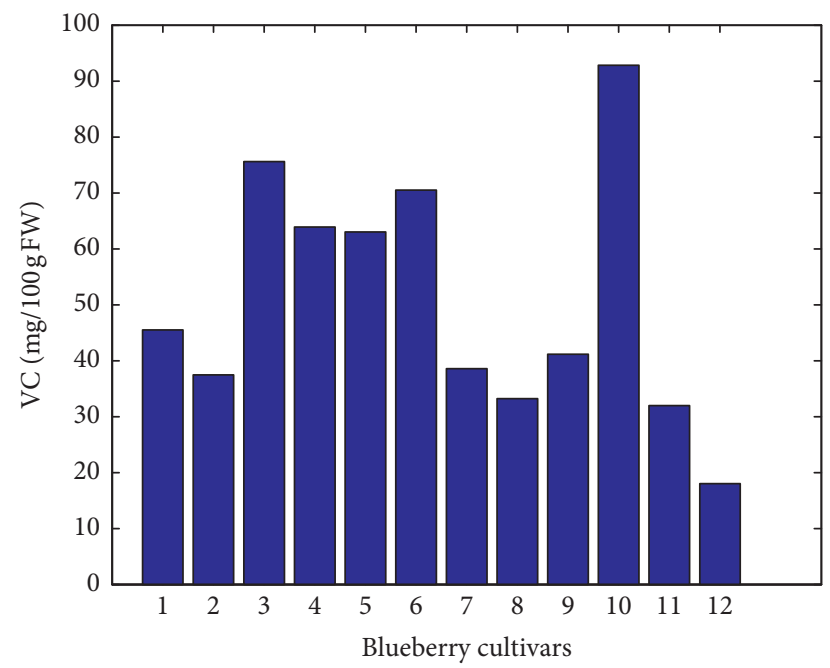

(b)

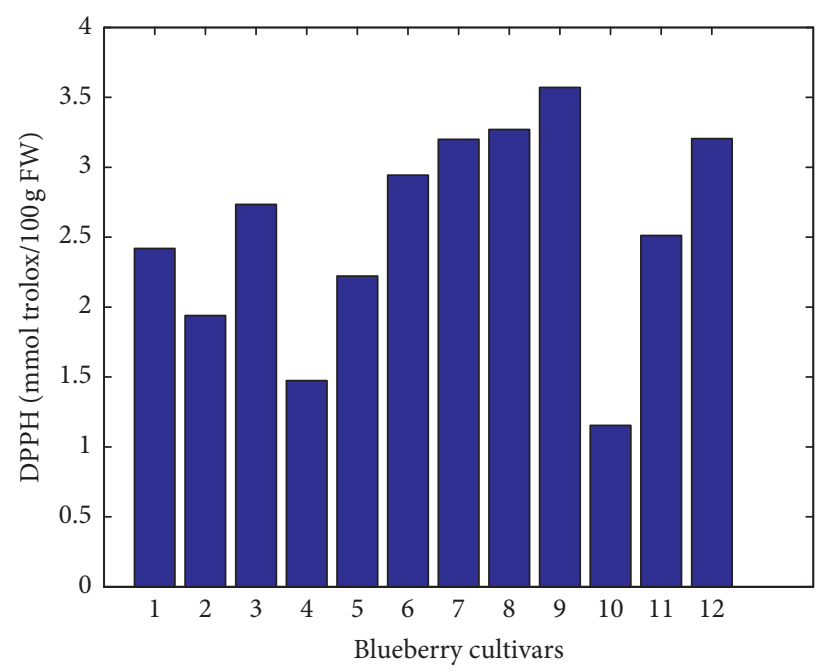

(c)

FIgURE 3: Averages of the 4 factors having the highest CV values over the 12 blueberries.

PLSDA, the 3 best subsets included 10,12, and 9 quality parameters, respectively, and for OVO-PLSDA, the 3 best subsets had 8,9 , and 8 quality parameters, respectively. Generally, OVO-PLSDA could obtain better training and prediction accuracy than OVR-PLSDA. This could be attributed to the large submodel complexity and uneven class sizes of OVR. Both OVR-PLSDA and OVO-PLSDA required at least 8 or 9 features to obtain best classification accuracy of the 12 blueberries, indicating that the variations among different blueberries were multivariate.

To demonstrate the variations in key quality factors, the pooled coefficient of variation $(\mathrm{CV})$ for each variable was computed and compared. The best classification accuracy of 0.915 was obtained by OVO-PLSDA using 8 selected quality parameters, including hardness (CV, 0.2593), juice yield (CV, 0.1516), titratable acidity (CV, 0.2692), vitamin 
C (CV, 0.4289), total phenols (CV, 0.1666), total flavonoids (CV, 0.1998), anthocyanins (CV, 0.2773), and antioxidant capacity (DPPH) (CV, 0.3137). Averages of the 4 factors having the highest $\mathrm{CV}$ values over the 12 blueberries are shown in Figure 3. shows that the correlation of titratable acidity and vitamin $\mathrm{C}$ was 0.64 and anthocyanins and $\mathrm{DPPH}$ was 0.56 . The low correlations among the key quality factors also imply that the discrimination of different blueberries requires multivariate quality factors. Key quality factors could be identified from their high frequency of being included in the selected sets in Table 1, including hardness (4), total flavonoids (6), vitamin C (5), total phenols (5), anthocyanins (6), and DPPH (5), indicating that all of the 3 types of quality factors are useful to characterize and discriminate different blueberries. The results demonstrated that the quality of blueberry is of multivariate nature, and the analysis requires the aiding of chemometrics.

\section{Conclusions}

In this study, the quality factors of blueberry fruits from 12 different cultivars in China were compared by analysis of 6 physical parameters, 12 chemical and nutritional components, and 3 antioxidant indices. Unsupervised PCA and supervised PLSDA were used to reveal the variations among different blueberries and to identify the key quality factors aided by the PSO algorithm. The results indicated that high classification accuracy (0.915) of the 12 blueberries could be obtained by using 8 quality factors, and all of the 3 types of quality factors are useful to characterize and discriminate different blueberries. Key quality factors were identified from their high frequency of being selected by PSO, including hardness, total flavonoids, vitamin C, total phenols, anthocyanins, and DPPH.

\section{Data Availability}

The data used to support the findings of this study are available from the corresponding author upon request.

\section{Conflicts of Interest}

The authors declare that they have no conflicts of interest.

\section{Authors' Contributions}

Juan Song and Qiong Shi equally contributed to this work.

\section{Acknowledgments}

Authors are grateful to the financial support from the $\mathrm{Na}$ tional Natural Science Foundation of China (Grants nos. 21665022, 31972164, 21776321, and 21706233), Key Projects of Technological Innovation of Hubei Province (2016ACA138), Guizhou Provincial Science and Technology Department (Nos. QKHJC[2017]1186, QKHZC[2019]2816, and QKHPTRC[2020]5009), the Talented Researcher Program from Guizhou Provincial Department of Education (QJHKYZ[2018]073 and QJHKYZ[2015]390), Tongren
Science and Technology Bureau (No. TSKY2019-3), the Talented Youth Cultivation Program from "the Fundamental Research Funds for the Central Universities", and South-Central University for Nationalities (No. CZP20007).

\section{References}

[1] M. E. Camire, "Bilberries and blueberries as functional foods and nutraceuticals," Bilberries and blueberries as functional foods and nutraceuticals," in Functional Foods: Herbs, Botanicals and Teas, G. Mazza and B. D. Oomah, Eds., vol. 5, no. 3, , pp. 289-319, Technomic Publishing, Lancaster, PA, USA, 2000.

[2] W. Routray and V. Orsat, "Blueberries and their anthocyanins: factors affecting biosynthesis and properties," Comprehensive Reviews in Food Science and Food Safety, vol. 10, no. 6, pp. 303-320, 2011.

[3] L. Ma, Z. Sun, Y. Zeng, M. Luo, and J. Yang, "Molecular mechanism and health role of functional ingredients in blueberry for chronic disease in human beings," International Journal of Molecular Sciences, vol. 19, no. 9, p. 2785, 2018.

[4] R. T. Hoskin, J. Xiong, D. A. Esposito, and M. A. Lila, "Blueberry polyphenol-protein food ingredients: the impact of spray drying on the in vitro antioxidant activity, anti-inflammatory markers, glucose metabolism and fibroblast migration," Food Chemistry, vol. 280, pp. 187-194, 2019.

[5] S. Y. Wang, M. J. Camp, and M. K. Ehlenfeldt, "Antioxidant capacity and $\alpha$-glucosidase inhibitory activity in peel and flesh of blueberry (Vaccinium spp.) cultivars," Food Chemistry, vol. 132, no. 4, pp. 1759-1768, 2012.

[6] M. S. Baliga and S. K. Katiyar, "Chemoprevention of photocarcinogenesis by selected dietary botanicals," Photochemical and Photobiological Sciences, vol. 5, no. 2, pp. 243-253, 2006.

[7] H. Tapiero, K. D. Tew, G. Mathé, and G. Mathe, "Polyphenols: do they play a role in the prevention of human pathologies?" Biomedicine \& Pharmacotherapy, vol. 56, no. 4, pp. 200-207, 2002.

[8] M. H. Grace, J. Xiong, D. Esposito, M. Ehlenfeldt, and M. A. Lila, "Simultaneous LC-MS quantification of anthocyanins and non-anthocyanin phenolics from blueberries with widely divergent profiles and biological activities," Food Chemistry, vol. 277, pp. 336-346, 2019.

[9] E. L. Boespflug, J. C. Eliassen, J. A. Dudley et al., "Enhanced neural activation with blueberry supplementation in mild cognitive impairment," Nutritional Neuroscience, vol. 21, no. 4, pp. 297-305, 2018.

[10] I. M. Heinonen, A. S. Meyer, and E. N. Frankel, "Antioxidant activity of berry phenolics on human low-density lipoprotein and liposome oxidation," Journal of Agricultural and Food Chemistry, vol. 46, no. 10, pp. 4107-4112, 1998.

[11] L. Zhu, X. Liu, J. Tan, and B. Wang, "Influence of harvest season on antioxidant activity and constituents of rabbiteye blueberry (Vaccinium ashei) leaves," Journal of Agricultural and Food Chemistry, vol. 61, no. 47, pp. 11477-11483, 2013.

[12] C. Li, J. Feng, W.-Y. Huang, and X.-T. An, "Composition of polyphenols and antioxidant activity of rabbiteye blueberry (Vaccinium ashei) in Nanjing," Journal of Agricultural and Food Chemistry, vol. 61, no. 3, pp. 523-531, 2013.

[13] C. Gentile, E. Di Gregorio, V. Di Stefano et al., "Food quality and nutraceutical value of nine cultivars of mango (Mangifera indica L.) fruits grown in Mediterranean subtropical environment," Food Chemistry, vol. 277, pp. 471-479, 2019. 
[14] R. Correia, M. H. Grace, D. Esposito, and M. A. Lila, "Wild blueberry polyphenol-protein food ingredients produced by three drying methods: comparative physico-chemical properties, phytochemical content, and stability during storage," Food Chemistry, vol. 235, no. 15, pp. 76-85, 2017.

[15] I. Sim, D. H. Suh, D. Singh et al., "Unraveling metabolic variation for blueberry and chokeberry cultivars harvested from different geo-climatic regions in Korea," Journal of Agricultural and Food Chemistry, vol. 65, no. 41, pp. 9031-9040, 2017.

[16] Z. Cai and W. Zhu, "Feature selection for multi-label classification using neighborhood preservation," IEEE/CAA Journal of Automatica Sinica, vol. 5, no. 1, pp. 320-330, 2018.

[17] H. Liu, M. Zhou, and Q. Liu, "An embedded feature selection method for imbalanced data classification," IEEE/CAA Journal of Automatica Sinica, vol. 6, no. 3, pp. 703-715, 2019.

[18] S. Gao, M. Zhou, Y. Wang, J. Cheng, H. Yachi, and J. Wang, "Dendritic Neuron model with effective learning algorithms for classification, approximation, and prediction," IEEE Transactions on Neural Networks and Learning Systems, vol. 30, no. 2, pp. 601-614, 2019.

[19] W. Dong and M. Zhou, "A supervised learning and control method to improve particle swarm optimization algorithms," IEEE Transactions on Systems, Man and Cybernetics: Systems, vol. 47, no. 7, pp. 1149-1159, 2017.

[20] P. Roy, G. S. Mahapatra, and K. M. Dey, "Forecasting of software reliability using neighborhood fuzzy particle swarm optimization based novel neural network," IEEE/CAA Journal of Automatica Sinica, vol. 6, no. 6, pp. 1365-1383, 2019.

[21] H. Han, M. Zhou, and Y. Zhang, "Can Virtual samples solve small sample size problem of KISSME in pedestrian Reidentification of smart transportation?" IEEE Transactions on Intelligent Transportation Systems, vol. 21, no. 9, pp. 3766-3776, 2019.

[22] N. Zhu, Y. Zhu, N. Yu et al., "Evaluation of microbial, physicochemical parameters and flavor of blueberry juice after microchip-pulsed electric field," Food Chemistry, vol. 274, pp. 146-155, 2019.

[23] N. Ghanei Ghooshkhaneh, M. R. Golzarian, and M. Mamarabadi, "Detection and classification of citrus green mold caused by Penicillium digitatum using multispectral imaging," Journal of the Science of Food and Agriculture, vol. 98, no. 9, pp. 3542-3550, 2018.

[24] K. Sabanci, A. Kayabasi, and A. Toktas, "Computer visionbased method for classification of wheat grains using artificial neural network," Journal of the Science of Food and Agriculture, vol. 97, no. 8, pp. 2588-2593, 2017.

[25] D. N. Li, X. J. Meng, and B. Li, "Profiling of anthocyanins from blueberries produced in China using HPLC-DAD-MS and exploratory analysis by principal component analysis," Journal of Food Composition and Analysis, vol. 47, pp. 1-7, 2015.

[26] F. Yuan, K. Cheng, J. Gao, and S. Pan, "Characterization of cultivar differences of blueberry wines using GC-QTOF-MS and metabolic profiling methods," Molecules, vol. 23, no. 9, p. $2376,2018$.

[27] C. S. W. Miaw, P. M. Santos, A. R. C. S. Silva et al., "Comparison of different multivariate classification methods for the detection of adulterations in grape Nectars by using low-field Nuclear magnetic resonance," Food Analytical Methods, vol. 13, no. 1, pp. 108-118, 2020.

[28] S. A. Bortolato, J. A. Arancibia, G. M. Escandar, and A. C. Olivieri, "Improvement of residual bilinearization by particle swarm optimization for achieving the second-order advantage with unfolded partial least-squares," Journal of Chemometrics, vol. 21, no. 12, pp. 557-566, 2007.

[29] M.-H. Hu, Q.-L. Dong, B.-L. Liu, and U. L. Opara, "Prediction of mechanical properties of blueberry using hyperspectral interactance imaging," Postharvest Biology and Technology, vol. 115, pp. 122-131, 2016.

[30] M. De Marchi, C. C. Fagan, C. P. O’Donnell et al., "Prediction of coagulation properties, titratable acidity, and $\mathrm{pH}$ of bovine milk using mid-infrared spectroscopy," Journal of Dairy Science, vol. 92, no. 1, pp. 423-432, 2009.

[31] T. Joyce, S. Y. Thompson, and J. Edwards-Webb, "Observations on the use of 2,4-dinitrophenylhydrazine and of 2,6dichlorophenolindophenol for the determination of vitamin $\mathrm{C}$ in raw and in heat-treated milk," Journal of Dairy Research, vol. 37, no. 1, pp. 29-45, 1970.

[32] V. L. Singleton and J. A. Rossi, "Colorimetry of total phenolics with phosphomolybdic-phosphotungstic acid reagents," American Journal of Enology and Viticulture, vol. 16, no. 3, pp. 144-158, 1965.

[33] Z. S. Jia, M. C. Tang, and J. M. Wu, "The determination of flavonoid contents in mulberry and their scavenging effects on superoxide radicals," Food Chemistry, vol. 64, no. 4, pp. 555-559, 1999.

[34] B. L. Somani, J. Khanade, and R. Sinha, "A modified anthronesulfuric acid method for the determination of fructose in the presence of certain proteins," Analytical Biochemistry, vol. 167, no. 2, pp. 327-330, 1987.

[35] H. T. S. Britton and L. Phillips, "The potentiometric titration of glucose with alkaline tartrate solutions of copper, including Fehling's solution," The Analyst, vol. 65, no. 766, pp. 18-24, 1940.

[36] A. Szumny, A. Figiel, A. Gutiérrez-Ortíz, and Á. A. CarbonellBarrachina, "Composition of rosemary essential oil (Rosmarinus officinalis) as affected by drying method," Journal of Food Engineering, vol. 97, no. 2, pp. 253-262, 2010.

[37] S. Han, S. Zou, X. He, K. Huang, and X. Mei, "Potential subchronic food safety of the stacked trait transgenic maize GH5112E-117C in Sprague-Dawley rats," Transgenic Research, vol. 25, no. 4, pp. 453-463, 2016.

[38] T. Yasuhara and K. Nokihara, "High-throughput analysis of total nitrogen content that replaces the classic Kjeldahl method," Journal of Agricultural and Food Chemistry, vol. 49, no. 10 , pp. 4581-4583, 2001.

[39] V. Lohachoompol, M. Mulholland, G. Srzednicki, and J. Craske, "Determination of anthocyanins in various cultivars of highbush and rabbiteye blueberries," Food Chemistry, vol. 111, no. 1, pp. 249-254, 2008.

[40] X. Su, J. Zhang, H. Wang et al., "Phenolic acid profiling, antioxidant, and anti-inflammatory activities, and miRNA regulation in the polyphenols of 16 blueberry samples from China," Molecules, vol. 22, no. 2, p. 312, 2017.

[41] V. Kraujalytė, P. R. Venskutonis, A. Pukalskas, L. Česonienė, and R. Daubaras, "Antioxidant properties, phenolic composition and potentiometric sensor array evaluation of commercial and new blueberry (Vaccinium corymbosum) and bog blueberry (Vaccinium uliginosum) genotypes," Food Chemistry, vol. 88, pp. 583-590, 2015.

[42] K. Sendin, M. Manley, V. Baeten, J. A. Fernández Pierna, and P. J. Williams, "Near infrared hyperspectral imaging for white maize classification according to grading regulations," Food Analytical Methods, vol. 12, no. 7, pp. 1612-1624, 2019.

[43] R. D. Snee, "Validation of regression models: methods and examples," Technometrics, vol. 19, no. 4, pp. 415-428, 1977. 
[44] M. Barker and W. Rayens, "Partial least squares for discrimination," Journal of Chemometrics, vol. 17, no. 3, pp. 166-173, 2003.

[45] S. Ghidini, M. O. Varrà, C. Dall'Asta, A. Badiani, A. Ianieri, and E. Zanardi, "Rapid authentication of European sea bass (Dicentrarchus labrax L.) according to production method, farming system, and geographical origin by near infrared spectroscopy coupled with chemometrics," Food Chemistry, vol. 280, pp. 321-327, 2019.

[46] S. Martín-Torres, A. M. Jiménez-Carvelo, A. GonzálezCasado, and L. Cuadros-Rodríguez, "Differentiation of avocados according to their botanical variety using liquid chromatographic fingerprinting and multivariate classification tree," Journal of the Science of Food and Agriculture, vol. 99, pp. 4932-4941, 2019.

[47] R. Moscetti, E. Radicetti, D. Monarca, M. Cecchini, and R. Massantini, "Near infrared spectroscopy is suitable for the classification of hazelnuts according to protected designation of origin," Journal of the Science of Food and Agriculture, vol. 95, no. 13, pp. 2619-2625, 2015.

[48] H.-Y. Fu, Q.-B. Yin, L. Xu et al., "Challenges of large-classnumber classification (LCNC): a novel ensemble strategy (ES) and its application to discriminating the geographical origins of 25 green teas," Chemometrics and Intelligent Laboratory Systems, vol. 157, pp. 43-49, 2016.

[49] R. Tierno, A. López, P. Riga et al., "Phytochemicals determination and classification in purple and red fleshed potato tubers by analytical methods and near infrared spectroscopy," Journal of the Science of Food and Agriculture, vol. 96, no. 6, pp. 1888-1899, 2016.

[50] Q.-S. Xu and Y.-Z. Liang, "Monte Carlo cross validation," Chemometrics and Intelligent Laboratory Systems, vol. 56, no. 1, pp. 1-11, 2001.

[51] M. Daszykowski, S. Serneels, K. Kaczmarek, P. Van Espen, C. Croux, and B. Walczak, "TOMCAT: a MATLAB toolbox for multivariate calibration techniques," Chemometrics and Intelligent Laboratory Systems, vol. 85, no. 2, pp. 269-277, 2007. 